\title{
Non-uniform Berry-Esseen Bounds for Weighted U-Statistics and Generalized L-Statistics
}

\author{
Haojun Hu • Qi-Man Shao
}

Received: 19 August 2013 / Accepted: 23 September 2013 / Published online: 22 November 2013 (C) School of Mathematical Sciences, University of Science and Technology of China and Springer-Verlag Berlin Heidelberg 2013

\begin{abstract}
Weighted U-statistics and generalized L-statistics are commonly used in statistical inference and their asymptotic properties have been well developed. In this paper sharp non-uniform Berry-Esseen bounds for weighted U-statistics and generalized L-statistic are established.
\end{abstract}

Keywords Weighted U-statistics · Generalized L-statistic · L-statistic • U-statistic Non-uniform Berry-Esseen bound · Normal approximation

Mathematics Subject Classification (2010) Primary 60F05 · Secondary 62E20

\section{Introduction and Main Results}

Let $\left\{X_{i}, 1 \leq i \leq n\right\}$ be a sequence of independent and identically distributed (i.i.d.) real-valued random variables and let $h\left(x_{1}, \ldots, x_{m}\right)$ be a kernel of degree $m$, that is, $h$ is a real-valued measurable symmetric function in $m$ arguments. The main purpose of this paper is to establish non-uniform Berry-Esseen bounds for weighted U-statistics and generalized L-statistics.

1.1 Weighted U-Statistics

Let $a: N^{m} \rightarrow R$ be a symmetric function. A weighted U-statistic is given by

$$
U_{n}=\sum_{1 \leq i_{1}<\cdots<i_{m} \leq n} a\left(i_{1}, \ldots, i_{m}\right) h\left(X_{i_{1}}, \ldots, X_{i_{m}}\right) .
$$

\footnotetext{
H. Hu

Department of Mathematics, Zhejiang University, Hangzhou, Zhejiang, China e-mail: huhaojun1989@foxmail.com

Q.-M. Shao $(\bowtie)$

Department of Statistics, The Chinese University of Hong Kong, Shatin, NT, Hong Kong, China e-mail: qmshao@sta.cuhk.edu.hk
} 
Clearly, it reduces to the Hoeffding U-statistics when $a \equiv 1$. The weighted U-statistic was first introduced by Shapiro and Hubert [10] for $m=2$ and its asymptotic normality and non-normality were discussed in O'Neil and Redner [8]. The results of O'Neil and Redner [8] were extended by Major [7] for general $m$. We refer to Rifi and Utzet [9] for the asymptotic behavior of the weighted U-statistics.

To present a non-uniform Berry-Esseen bound for the weighted U-statistic, assume

$$
E h\left(X_{1}, \ldots, X_{m}\right)=0, \quad E h^{2}\left(X_{1}, \ldots, X_{m}\right)<\infty
$$

Put

$$
\begin{aligned}
\sigma_{0}^{2} & =E h^{2}\left(X_{1}, \ldots, X_{m}\right)<\infty, \quad g_{1}(x)=E\left[h\left(X_{1}, \ldots, X_{m}\right) \mid X_{1}=x\right], \\
\sigma_{1}^{2} & =E g_{1}^{2}\left(X_{1}\right), \\
A_{l} & =\sum_{\substack{1 \leq i_{1}<\ldots<i_{m-1} \leq n, i_{j} \neq l, j \in\{1, \ldots, m-1\}}} a\left(i_{1}, \ldots, i_{m-1}, l\right), \\
B_{k} & =\left|\sum_{\left.\left(i_{1}, \ldots, i_{m}\right),\left(j_{1}, \ldots, j_{m}\right)\right) \in \mathcal{H}_{k}} a\left(i_{1}, \ldots, i_{m}\right) a\left(j_{1}, \ldots, j_{m}\right)\right|,
\end{aligned}
$$

and

$$
C_{k, l}=\left|\sum_{\left.\left(i_{1}, \ldots, i_{m-1}\right),\left(j_{1}, \ldots, j_{m-1}\right)\right) \in \mathcal{H}_{k, l}} a\left(i_{1}, \ldots, i_{m-1}, l\right) a\left(j_{1}, \ldots, j_{m-1}, l\right)\right|,
$$

where $\mathcal{H}_{k}$ denotes the set of $1 \leq i_{1}<\cdots<i_{m} \leq n, 1 \leq j_{1}<\cdots<j_{m} \leq n$ with $k$ common elements between the two sets $\left\{i_{1}, \ldots, i_{m}\right\}$ and $\left\{j_{1}, \ldots, j_{m}\right\}$, and $\mathcal{H}_{k, l}$ denotes the set of $1 \leq i_{1}<\cdots<i_{m-1} \leq n, 1 \leq j_{1}<\cdots<j_{m-1} \leq n, i_{s} \neq l, j_{s} \neq l$ for each $1 \leq s \leq m-1$ with $k$ common elements between the two sets $\left\{i_{1}, \ldots, i_{m-1}\right\}$ and $\left\{j_{1}, \ldots, j_{m-1}\right\}$.

The following theorem gives a non-uniform Berry-Esseen bound for $U_{n}$.

Theorem 1.1 If $E h\left(X_{1}, \ldots, X_{m}\right)=0$ and $E\left|g_{1}\left(X_{1}\right)\right|^{p}<\infty$ for $2<p \leq 3$, then there exists a universal constant $C$ such that

$$
\begin{aligned}
& \left|P\left(\frac{U_{n}}{\left(\sum_{j=1}^{n} A_{j}^{2}\right)^{1 / 2} \sigma_{1}} \leq z\right)-\Phi(z)\right| \\
& \leq \frac{9 \sigma_{0}^{2} \sum_{k=2}^{m} k B_{k}}{m(|z|+1)^{2} \sigma_{1}^{2} \sum_{j=1}^{n} A_{j}^{2}}+\frac{C}{(|z|+1)^{p}}\left(\frac{\sigma_{0}\left(\sum_{k=2}^{m} k B_{k}\right)^{1 / 2}}{\sigma_{1}\left(m \sum_{j=1}^{n} A_{j}^{2}\right)^{1 / 2}}\right. \\
& \left.\quad+\frac{\sigma_{0}\left(\sum_{l=1}^{n}\left|A_{l}\right|\left(\sum_{k=2}^{m} k C_{k-1, l}\right)^{1 / 2}\right)}{\sigma_{1} m^{1 / 2} \sum_{j=1}^{n} A_{j}^{2}}+\frac{\left.E\left|g\left(X_{1}\right)^{p} \sum_{j=1}^{n}\right| A_{j}\right|^{p}}{\sigma_{1}^{p}\left(\sum_{j=1}^{n} A_{j}^{2}\right)^{p / 2}}\right) .
\end{aligned}
$$

Remark 1.1 It is easy to see that when $a \equiv 1$,

$$
\begin{aligned}
A_{l} & =\frac{m}{n}\left(\begin{array}{c}
n \\
m
\end{array}\right), \quad B_{k}=\left(\begin{array}{c}
n \\
m
\end{array}\right)\left(\begin{array}{c}
m \\
k
\end{array}\right)\left(\begin{array}{l}
n-m \\
m-k
\end{array}\right), \\
C_{k, l} & =\left(\begin{array}{c}
n-1 \\
m-1
\end{array}\right)\left(\begin{array}{c}
m-1 \\
k
\end{array}\right)\left(\begin{array}{c}
n-m \\
m-1-k
\end{array}\right) .
\end{aligned}
$$


Result (1.7) recovers Theorem 3.1 of Chen and Shao [4].

Remark 1.2 For $m=2$ letting $a(l, l)=0$, we have

$$
A_{l}=\sum_{j=1}^{n} a(j, l), \quad B_{2}=\sum_{1 \leq i<j \leq n} a^{2}(i, j)
$$

and

$$
C_{1, l}=\sum_{j=1}^{n} a^{2}(j, l)
$$

So the non-uniform Berry-Esseen bound (1.7) is reduced to

$\frac{9 \sigma_{0}^{2} B_{2}}{(|z|+1)^{2} \sigma_{1}^{2} \sum_{j=1}^{n} A_{j}^{2}}+\frac{C}{(|z|+1)^{p}}\left(\frac{\sigma_{0} B_{2}^{1 / 2}}{\sigma_{1}\left(\sum_{j=1}^{n} A_{j}^{2}\right)^{1 / 2}}+\frac{E\left|g\left(X_{1}\right)\right|^{p} \sum_{j=1}^{n}\left|A_{j}\right|^{p}}{\sigma_{1}^{p}\left(\sum_{j=1}^{n} A_{j}^{2}\right)^{p / 2}}\right)$.

In particular, if

$$
\left(B_{2}+\max _{1 \leq i \leq n} A_{i}^{2}\right) / \sum_{j=1}^{n} A_{j}^{2} \rightarrow 0,
$$

then $U_{n} /\left(\sum_{j=1}^{n} A_{j}^{2}\right)^{1 / 2} \sigma_{1}$ is asymptotically normal.

\subsection{Generalized L-Statistics}

Let $R_{n, 1} \leq \cdots \leq R_{n, C_{n, m}}$ be the order values of $h\left(X_{i_{1}}, \ldots, X_{i_{m}}\right)$ taken over $m$-tuples $\left\{\left(i_{1}, \ldots, i_{m}\right): 1 \leq i_{1}<\cdots<i_{m} \leq n\right\}$, where $C_{n, m}=\left(\begin{array}{c}n \\ m\end{array}\right)$. A generalized L-statistic is defined by

$$
T_{n}=\sum_{i=1}^{C_{n, m}} c_{n, i} R_{n, i}
$$

where

$$
c_{n, i}=\int_{(i-1) / C_{n, m}}^{i / C_{n, m}} J(t) d t
$$

and $J$ is a weight function on $[0,1]$. The generalized L-statistic was first introduced by Serfling [12]. It includes Hodges-Lehmann location estimator, $U$-statistics, trimmed $U$-statistics, Winsorized $U$-statistics as special cases. The asymptotic properties of the generalized L-statistics have been extensively studied. We refer to Serfling [12, 13] Borovshikh and Weber [1] for the asymptotic normality and for the general theory and applications, Cai [2] for the moderate and large deviations, and Helmers and Ruymgaart [6], Helmers, Jansseen and Serfling [5] for the uniform Berry-Esseen bounds.

Let $H(y)$ be the distribution function of the random variable function $h\left(X_{1}, \ldots, X_{m}\right)$ and denote the empirical distribution function by

$$
H_{n}(y)=\left(\begin{array}{c}
n \\
m
\end{array}\right)^{-1} \sum_{1 \leq i_{1}<\cdots<i_{m} \leq n} I\left(h\left(X_{i_{1}}, \ldots, X_{i_{m}}\right) \leq y\right), \quad y \in R .
$$


Let $T$ be an L-functional

$$
T(G)=\int_{0}^{1} J(t) G^{-1}(t) d t
$$

with $G^{-1}(t)=\inf \{y: G(y) \geq t\}$ for any distribution function $G$. Clearly,

$$
T_{n}=T\left(H_{n}\right)
$$

and the generalized L-statistic $T_{n}$ is actually estimating the parameter $T(H)$.

Let

$$
\begin{aligned}
A\left(x_{1}, \ldots, x_{m}\right) & =\int_{-\infty}^{+\infty}\left(I\left(h\left(x_{1}, \ldots, x_{m}\right) \leq y\right)-H(y)\right) J(H(y)) d y, \\
g_{2}(x) & =E\left(A\left(X_{1}, \ldots, X_{m}\right) \mid X_{1}=x\right) .
\end{aligned}
$$

Put

$$
\sigma_{0}^{2}=E h^{2}\left(X_{1}, \ldots, X_{m}\right), \quad \sigma_{2}^{2}=\operatorname{Var}\left(g_{2}\left(X_{1}\right)\right) .
$$

Then we have the following non-uniform Berry-Esseen bound for $T_{n}$.

Theorem 1.2 Assume that $1 \leq m \leq n / 5$ and there exists a constant $c_{0}$ such that

$$
|J(t)-J(s)| \leq c_{0}|t-s| \text { for } 0 \leq s, t \leq 1 .
$$

If $E h\left(X_{1}, \ldots, X_{m}\right)=0$ and $E\left|g_{2}\left(X_{1}\right)\right|^{p}<\infty$ for $2<p \leq 3$, then there exists $a$ universal constant $C$ such that

$$
\begin{aligned}
\mid P( & \left.\frac{\sqrt{n}}{m \sigma_{2}}\left(T\left(H_{n}\right)-T(H)\right) \leq z\right)-\Phi(z) \mid \\
\leq & \frac{36 m \sigma_{0}^{2}\left(\left(|J(0)|+c_{0}\right)^{2}+6 m c_{0}^{2}\right)}{n \sigma_{2}^{2}(|z|+1)^{2}} \\
& \quad+\frac{C}{(|z|+1)^{p}}\left(\frac{\left(|J(0)|+c_{0}\right) m \sigma_{0}}{n^{1 / 2} \sigma_{2}}+\frac{E\left|g_{2}\left(X_{1}\right)\right|^{p}}{n^{(p-2) / 2} \sigma_{2}^{p}}\right) .
\end{aligned}
$$

Theorem 1.2 recovers the uniform Berry-Esseen bound of Helmers, Janssen and Serfling [5] with a smaller order in terms of $m$.

\section{Proofs}

Our proof is based on the following non-uniform Berry-Esseen inequality of Chen and Shao [4]:

Proposition 2.1 Let $\xi_{1}, \ldots, \xi_{n}$ be independent random variables satisfying $E \xi_{i}=0$ for $i=1, \ldots, n$, and $\sum_{i=1}^{n} E\left(\xi_{i}^{2}\right)=1$. Let $W=\sum_{i=1}^{n} \xi_{i}$ and $\Delta$ be a measurable function of $\left\{\xi_{i}, 1 \leq i \leq n\right\}$. If $E\left|\xi_{i}\right|^{p}<\infty$ for some $2<p \leq 3$, then there exists an absolute constant $C$ such that 


$$
\begin{aligned}
& |P(W+\Delta \leq z)-\Phi(z)| \\
& \quad \leq \frac{9\|\Delta\|_{2}^{2}}{(1+|z|)^{2}}+\frac{C}{(|z|+1)^{p}}\left(\|\Delta\|_{2}+\sum_{i=1}^{n}\left\|\xi_{i}\right\|_{2}\left\|\Delta-\Delta_{i}\right\|_{2}+\sum_{i=1}^{n} E\left|\xi_{i}\right|^{p}\right)
\end{aligned}
$$

where $\Delta_{i}$ is any random variable such that $\xi_{i}$ and $\left(W-\xi_{i}, \Delta_{i}\right)$ are independent, and $\|\xi\|_{2}$ denotes $\left(E \xi^{2}\right)^{1 / 2}$.

\subsection{Proof of Theorem 1.1}

To apply Proposition 2.1, the first step is to rewrite

$$
\frac{1}{\left(\sum_{j=1}^{n} A_{j}^{2}\right)^{1 / 2} \sigma_{1}} U_{n}=W+\Delta,
$$

where $W$ is a sum of independent random variables and $\Delta$ is a negligible term. For $1 \leq k \leq m$, let

$$
\begin{aligned}
& h_{k}\left(x_{1}, \ldots, x_{k}\right)=E\left(h\left(X_{1}, \ldots, X_{m}\right) \mid X_{1}=x_{1}, \ldots, X_{k}=x_{k}\right), \\
& \bar{h}_{k}\left(x_{1}, \ldots, x_{k}\right)=h_{k}\left(x_{1}, \ldots, x_{k}\right)-\sum_{i=1}^{k} g_{1}\left(x_{i}\right), \\
& \bar{h}\left(x_{1}, \ldots, x_{m}\right)=\bar{h}_{m}\left(x_{1}, \ldots, x_{m}\right)=h\left(x_{1}, \ldots, x_{m}\right)-\sum_{j=1}^{m} g_{1}\left(x_{j}\right) .
\end{aligned}
$$

Then we can take

$$
W=\sum_{i=1}^{n} \xi_{i}, \quad \xi_{i}=\frac{A_{j}}{\left(\sum_{j=1}^{n} A_{j}^{2}\right)^{1 / 2} \sigma_{1}} g_{1}\left(X_{j}\right),
$$

and

$$
\Delta=\frac{1}{\left(\sum_{j=1}^{n} A_{j}^{2}\right)^{1 / 2} \sigma_{1}} \sum_{1 \leq i_{1}<\cdots<i_{m} \leq n} a\left(i_{1}, \ldots, i_{m}\right) \bar{h}\left(X_{i_{1}}, \ldots, X_{i_{m}}\right) .
$$

Clearly, $\xi_{1}, \ldots, \xi_{n}$ are independent random variable with $E \xi_{i}=0$ and $\sum_{i=1}^{n} E\left(\xi_{i}^{2}\right)=1$. Set

$$
\Delta_{l}=\frac{1}{\left(\sum_{j=1}^{n} A_{j}^{2}\right)^{1 / 2} \sigma_{1}} \sum_{\substack{1 \leq i_{1}<\cdots<i_{m} \leq n, i_{j} \neq l, j \in\{1, \ldots, m\}}} a\left(i_{1}, \ldots, i_{m}\right) \bar{h}\left(X_{i_{1}}, \ldots, X_{i_{m}}\right) .
$$

Now by Proposition 2.1, Theorem 1.1 follows by the next two lemmas.

Lemma 2.1 We have

$$
E \Delta^{2} \leq \frac{\sigma_{0}^{2} \sum_{k=2}^{m} k B_{k}}{m \sigma_{1}^{2} \sum_{j=1}^{n} A_{j}^{2}}
$$


Lemma 2.2 We have

$$
E\left(\Delta-\Delta_{l}\right)^{2} \leq \frac{\sigma_{0}^{2} \sum_{k=2}^{m} k C_{k-1, l}}{m \sigma_{1}^{2} \sum_{j=1}^{n} A_{j}^{2}} .
$$

Proof of Lemma 2.1 Observe that

$$
\begin{aligned}
E \Delta^{2}= & \frac{1}{\sum_{j=1}^{n} A_{j}^{2} \sigma_{1}^{2}} E\left(\sum_{1 \leq i_{1}<\cdots<i_{m} \leq n} a\left(i_{1}, \ldots, i_{m}\right) \bar{h}\left(X_{i_{1}}, \ldots, X_{i_{m}}\right)\right)^{2} \\
= & \frac{1}{\sum_{j=1}^{n} A_{j}^{2} \sigma_{1}^{2}} \sum_{k=2}^{m}\left(\sum_{\left(\left(i_{1}, \ldots, i_{m}\right),\left(j_{1}, \ldots, j_{m}\right)\right) \in \mathcal{H}_{k}} a\left(i_{1}, \ldots, i_{m}\right) a\left(j_{1}, \ldots, j_{m}\right)\right) \\
& \times E \bar{h}_{k}^{2}\left(X_{1}, \ldots, X_{k}\right) \\
= & \frac{1}{\sum_{j=1}^{n} A_{j}^{2} \sigma_{1}^{2}} \sum_{k=2}^{m} B_{k} E \bar{h}_{k}^{2}\left(X_{1}, \ldots, X_{k}\right) .
\end{aligned}
$$

By Eq. (10.80) in [3], we have

$$
E \bar{h}_{k}^{2}\left(X_{1}, \ldots, X_{k}\right) \leq \frac{k}{m} \sigma_{0}^{2}
$$

and hence (2.2) holds.

Proof of Lemma 2.2 Similarly, we have

$$
\begin{aligned}
\sigma_{1}^{2}\left(\sum_{j=1}^{n} A_{j}^{2}\right) E\left(\Delta-\Delta_{l}\right)^{2} \\
=E\left(\left\{\sum_{1 \leq i_{1}<\cdots<i_{m} \leq n}-\sum_{\substack{1 \leq i_{1}<\cdots<i_{m} \leq n, i_{j} \neq l, j \in\{1, \ldots, m\}}}\right\} a\left(i_{1}, \ldots, i_{m}\right) \bar{h}\left(X_{i_{1}}, \ldots, X_{i_{m}}\right)\right)^{2} \\
=\sum_{k=1}^{m-1}\left(\sum_{\left(\left(i_{1}, \ldots, i_{m-1}\right),\left(j_{1}, \ldots, j_{m-1}\right)\right) \in \mathcal{H}_{k, l}} a\left(i_{1}, \ldots, i_{m-1}, l\right) a\left(j_{1}, \ldots, j_{m-1}, l\right)\right) \\
\quad \times E \bar{h}_{k+1}^{2}\left(X_{1}, \ldots, X_{k}, X_{n}\right) \\
=\sum_{k=1}^{m-1} C_{k, l} E \bar{h}_{k+1}^{2}\left(X_{1}, \ldots, X_{k}, X_{n}\right) .
\end{aligned}
$$

By Eq. (10.80) in [3] again, we have

$$
E \bar{h}_{k+1}^{2}\left(X_{1}, \ldots, X_{k}, X_{n}\right) \leq \frac{k+1}{m} \sigma_{0}^{2}
$$

and hence (2.3) holds. 


\subsection{Proof of Theorem 1.2}

As in the proof of Theorem 1.1, the first step is to rewrite $\sqrt{n}\left(T\left(H_{n}\right)-T(H)\right) /\left(m \sigma_{2}\right)$ as $W+\Delta$, where $W$ is a sum of independent random variables and $\Delta$ is negligible. To this end, let $A\left(x_{1}, \ldots, x_{m}\right)$ and $g_{2}(x)$ be defined as in (1.11) and (1.12) and put

$$
\bar{A}_{m}\left(x_{1}, \ldots, x_{m}\right)=A\left(x_{1}, \ldots, x_{m}\right)-\sum_{j=1}^{m} g_{2}\left(x_{j}\right) .
$$

Let $\varphi(t)=\int_{0}^{t} J(s) d s$. Observing that by the integration by parts (see, e.g., $[11$, p. 265])

$$
T\left(H_{n}\right)-T(H)=-\int_{-\infty}^{+\infty}\left(\varphi\left(H_{n}(y)\right)-\varphi(H(y))\right) d y,
$$

we have

$$
\frac{\sqrt{n}}{m \sigma_{2}}\left(T\left(H_{n}\right)-T(H)\right)=W+\Delta,
$$

where

$$
W=\sum_{i=1}^{n} \xi_{i}, \quad \xi_{i}=-g_{2}\left(X_{i}\right) /\left(\sqrt{n} \sigma_{2}\right)
$$

and

$$
\begin{aligned}
\Delta= & -\left(\begin{array}{c}
n \\
m
\end{array}\right)^{-1} \frac{\sqrt{n}}{m \sigma_{2}} \sum_{1 \leq i_{1}<\cdots<i_{m} \leq n} \bar{A}_{m}\left(x_{i_{1}}, \ldots, x_{i_{m}}\right) \\
& -\frac{\sqrt{n}}{m \sigma_{2}} \int_{-\infty}^{+\infty}\left[\varphi\left(H_{n}(y)\right)-\varphi(H(y))-\left(H_{n}(y)-H(y)\right) J(H(y))\right] d y .
\end{aligned}
$$

Clearly, $\xi_{1}, \ldots, \xi_{n}$ are i.i.d. random variable with $E \xi_{i}=0$ and $\sum_{i=1}^{n} E\left(\xi_{i}^{2}\right)=1$. Set

$$
\begin{aligned}
\Delta_{l}= & -\left(\begin{array}{c}
n \\
m
\end{array}\right)^{-1} \frac{\sqrt{n}}{m \sigma_{2}} \sum_{\substack{1 \leq i_{1}<\cdots<i_{m} \leq n, i_{j} \neq l, j \in\{1, \ldots, m\}}} \bar{A}_{m}\left(x_{i_{1}}, \ldots, x_{i_{m}}\right) \\
& -\frac{\sqrt{n}}{m \sigma_{2}} \int_{-\infty}^{+\infty}\left[\varphi\left(H_{n, l}(y)\right)-\varphi(H(y))-\left(H_{n, l}(y)-H(y)\right) J(H(y))\right] d y,
\end{aligned}
$$

where

$$
\begin{aligned}
& H_{n, l}(y) \\
& \quad=\left(\begin{array}{c}
n \\
m
\end{array}\right)^{-1}\left(\sum_{\substack{1 \leq i_{1}<\cdots<i_{m} \leq n, i_{j} \neq l, j \in\{1, \ldots, m\}}}\left(I\left[h\left(X_{i_{1}}, \ldots, X_{i_{m}}\right) \leq y\right]+\left(\begin{array}{c}
n-1 \\
m-1
\end{array}\right) H(y)\right)\right) .
\end{aligned}
$$

Now by Proposition 2.1, Theorem 1.2 follows by the next two lemmas. 
Lemma 2.3 We have

$$
E \Delta^{2} \leq \frac{4\left(\left(|J(0)|+c_{0}\right)^{2}+6 m c_{0}^{2}\right) m \sigma_{0}^{2}}{n \sigma_{2}^{2}} .
$$

Lemma 2.4 We have

$$
E\left(\Delta-\Delta_{l}\right)^{2} \leq \frac{16 m^{2}\left(|J(0)|+8 c_{0}\right)^{2} \sigma_{0}^{2}}{n^{2} \sigma_{2}^{2}} .
$$

The proofs of the two lemmas are presented in the next two subsections.

\subsubsection{Proof of Lemma 2.3}

Let $\sigma^{2}=E A^{2}\left(X_{1}, \ldots, X_{m}\right)$. We first show that

$$
\sigma^{2} \leq\left(|J(0)|+c_{0}\right)^{2} \sigma_{0}^{2} .
$$

Let $\xi=h\left(X_{1}, \ldots, X_{m}\right)$ and $\eta$ be an independent copy of $\xi$. Then

$$
\begin{aligned}
\sigma^{2} & =E \int_{-\infty}^{\infty} \int_{-\infty}^{\infty}(I(\xi \leq x)-H(x)) J(H(x))(I(\xi \leq y)-H(y)) J(H(y)) d x d y \\
& =\int_{-\infty}^{\infty} \int_{-\infty}^{\infty}(H(x \wedge y)-H(x) H(y)) J(H(x)) J(H(y)) d x d y \\
& \leq\left(|J(0)|+c_{0}\right)^{2} \int_{-\infty}^{\infty} \int_{-\infty}^{\infty}(H(x \wedge y)-H(x) H(y)) d x d y
\end{aligned}
$$

by the fact that (1.14) implies $|J(t)| \leq\left(|J(0)|+c_{0}\right)$ for $0 \leq t \leq 1$. Also note that

$$
\begin{aligned}
& \int_{-\infty}^{\infty} \int_{-\infty}^{\infty}(H(x \wedge y)-H(x) H(y)) d x d y \\
& \quad=2 \int_{-\infty}^{\infty} \int_{x \leq y} H(x)(1-H(y)) d x d y \\
& =2 E \int_{-\infty}^{\infty} \int_{x \leq y} I(\xi \leq x)(1-I(\eta \leq y)) d x d y \\
& =2 E \int_{\xi \leq x<y<\eta} I(\xi \leq x) I(y<\eta) d x d y \\
& =E(\eta-\xi)^{2} I(\xi<\eta)=(1 / 2) E(\xi-\eta)^{2} \\
& \leq E \xi^{2}=\sigma_{0}^{2} .
\end{aligned}
$$

This proves (2.12).

Let

$$
\begin{aligned}
h_{1}\left(x_{1}, \ldots, x_{2 m}\right)= & \int_{-\infty}^{\infty}\left\{I\left(h\left(x_{1}, \ldots, x_{m}\right) \leq y\right)-H(y)\right\} \\
& \times\left\{I\left(h\left(x_{m+1}, \ldots, x_{2 m}\right) \leq y\right)-H(y)\right\} d y
\end{aligned}
$$


and $\sigma_{3}^{2}=E h_{1}^{2}\left(X_{1}, \ldots, X_{2 m}\right)$. Then

$$
\sigma_{3}^{2} \leq \sigma_{0}^{2}
$$

In fact, by the Hölder inequality

$$
\begin{aligned}
\sigma_{3}^{2} \leq & E\left(\int_{-\infty}^{\infty}\left(I\left(h\left(X_{1}, \ldots, X_{m}\right) \leq y\right)-H(y)\right)^{2} d y\right. \\
& \left.\times \int_{-\infty}^{\infty}\left(I\left(h\left(X_{m+1}, \ldots, X_{2 m}\right) \leq y\right)-H(y)\right)^{2} d y\right) \\
= & \left(\int_{-\infty}^{\infty} H(y)(1-H(y)) d y\right)^{2} \\
\leq & \left(E\left|h\left(X_{1}, \ldots, X_{m}\right)\right|\right)^{2} \leq E h^{2}\left(X_{1}, \ldots, X_{m}\right)=\sigma_{0}^{2},
\end{aligned}
$$

as desired.

Next we obtain a similar result for $E h_{1}^{2}\left(X_{1}, \ldots, X_{m}, Y_{1}, \ldots, Y_{m}\right)$, where $\left(Y_{1}, \ldots Y_{m}\right)$ has the same distribution as that of $\left(X_{1}, \ldots, X_{m}\right)$. Let $\xi=h\left(X_{1}, \ldots, X_{m}\right)$ and $\eta=h\left(Y_{1}, \ldots, Y_{m}\right)$. Then

$$
\begin{aligned}
E & h_{1}^{2}\left(X_{1}, \ldots, X_{m}, Y_{1}, \ldots, Y_{m}\right) \\
& =E\left(\int_{-\infty}^{\infty}(I(\xi \leq x)-H(x))(I(\eta \leq x)-H(x)) d x\right)^{2} \\
& \leq 0.25 E\left(\int_{-\infty}^{\infty}(I(\xi \leq x)-H(x))^{2}+(I(\eta \leq x)-H(x))^{2} d x\right)^{2} \\
& \leq E\left(\int_{-\infty}^{\infty}(I(\xi \leq x)-H(x))^{2} d x\right)^{2} \\
& =\int_{-\infty}^{\infty} \int_{-\infty}^{\infty} E(I(\xi \leq x)-H(x))^{2}(I(\xi \leq y)-H(y))^{2} d x d y .
\end{aligned}
$$

A direct calculation shows that for $x \leq y$

$$
\begin{aligned}
E( & I(\xi \leq x)-H(x))^{2}(I(\xi \leq y)-H(y))^{2} \\
= & H(x)(1-2 H(x))(1-2 H(y))+H(x) H^{2}(y)(1-2 H(x)) \\
& +H^{2}(x) H(y)(1-2 H(y))+H^{2}(x) H^{2}(y) \\
= & H(x)\left(1-2 H(x)-2 H(y)+5 H(x) H(y)-3 H(x) H^{2}(y)+H^{2}(y)\right) \\
\leq & H(x)\left(1-H(y)+3 H(x) H(y)-3 H(x) H^{2}(y)\right) \\
= & H(x)(1-H(y))(1+3 H(x) H(y)) \\
\leq & 4 H(x)(1-H(y)) .
\end{aligned}
$$

Thus by (2.13), we have

$$
E h_{1}^{2}\left(X_{1}, \ldots, X_{m}, Y_{1}, \ldots, Y_{m}\right) \leq 4 E \xi^{2}=4 \sigma_{0}^{2} .
$$

To estimate $E \Delta^{2}$, write

$$
\Delta=A+B
$$


where

$$
\begin{aligned}
A & =-\left(\begin{array}{c}
n \\
m
\end{array}\right)^{-1} \frac{\sqrt{n}}{m \sigma_{2}} \sum_{1 \leq i_{1}<\cdots<i_{m} \leq n} \bar{A}_{m}\left(x_{i_{1}}, \ldots, x_{i_{m}}\right), \\
B & =-\frac{\sqrt{n}}{m \sigma_{2}} \int_{-\infty}^{+\infty}\left[\varphi\left(H_{n}(y)\right)-\varphi(H(y))-\left(H_{n}(y)-H(y)\right) J(H(y))\right] d y .
\end{aligned}
$$

Clearly,

$$
E \Delta^{2} \leq 2 E\left(A^{2}+B^{2}\right) .
$$

Following the proof in [3, pp. 284-286], we have

$$
\begin{aligned}
& E\left(\sum_{1 \leq i_{1}<\cdots<i_{m} \leq n} \bar{A}_{m}\left(x_{i_{1}}, \ldots, x_{i_{m}}\right)\right)^{2} \\
& \quad=\left(\begin{array}{c}
n \\
m
\end{array}\right) \sum_{j=2}^{m}\left(\begin{array}{c}
m \\
j
\end{array}\right)\left(\begin{array}{l}
n-m \\
m-j
\end{array}\right) E \bar{A}_{j}^{2}\left(X_{1}, \ldots, X_{n}\right) \\
& \quad \leq \frac{m(m-1)^{2}}{n(n-m+1)}\left(\begin{array}{l}
n \\
m
\end{array}\right)^{2} \sigma^{2}
\end{aligned}
$$

and hence

$$
\begin{aligned}
E A^{2} & \leq\left(\left(\begin{array}{c}
n \\
m
\end{array}\right)^{-1} \frac{\sqrt{n}}{m \sigma_{2}}\right)^{2} \frac{m(m-1)^{2}}{n(n-m+1)}\left(\begin{array}{l}
n \\
m
\end{array}\right)^{2} \sigma^{2} \\
& \leq \frac{(m-1)^{2} \sigma^{2}}{m(n-m+1) \sigma_{2}^{2}} \\
& \leq \frac{2 m\left(|J(0)|+c_{0}\right)^{2} \sigma_{0}^{2}}{n \sigma_{2}^{2}} .
\end{aligned}
$$

To finish the proof of (2.10) by (2.17) and (2.18), it suffices to show that

$$
E B^{2} \leq \frac{12 m^{2} c_{0}^{2} \sigma_{0}^{2}}{n \sigma_{2}^{2}}
$$

Recall that

$$
E B^{2}=\frac{n}{m^{2} \sigma_{2}^{2}} E\left(\int_{-\infty}^{+\infty}\left[\varphi\left(H_{n}(y)\right)-\varphi(H(y))-\left(H_{n}(y)-H(y)\right) J(H(y))\right] d y\right)^{2} .
$$

By the definition of $\varphi(t)$ and the Lipschitz condition (1.14)

$$
|\varphi(t)-\varphi(s)-(t-s) J(s)|=\left|\int_{0}^{t-s}(J(u+s)-J(s)) d u\right| \leq 0.5 c_{0}(t-s)^{2},
$$

we have 


$$
\begin{aligned}
& E\left(\int_{-\infty}^{+\infty}\left[\varphi\left(H_{n}(y)\right)-\varphi(H(y))-\left(H_{n}(y)-H(y)\right) J(H(y))\right] d y\right)^{2} \\
& \quad \leq 0.25 c_{0}^{2} E\left(\int_{-\infty}^{+\infty}\left(H_{n}(y)-H(y)\right)^{2} d y\right)^{2} .
\end{aligned}
$$

Write

$$
\int_{-\infty}^{+\infty}\left(H_{n}(y)-H(y)\right)^{2} d y=Q_{n}+R_{n}
$$

where

$$
\begin{aligned}
Q_{n} & =\left(\begin{array}{c}
n \\
m
\end{array}\right)^{-2} \sum_{1}^{*} h_{1}\left(X_{i_{1}}, \ldots, X_{i_{m}}, X_{j_{1}} \ldots, X_{j_{m}}\right) \\
R_{n} & =\left(\begin{array}{c}
n \\
m
\end{array}\right)^{-2} \sum_{1}^{* *} h_{1}\left(X_{i_{1}}, \ldots, X_{i_{m}}, X_{j_{1}} \ldots, X_{j_{m}}\right),
\end{aligned}
$$

here $\sum_{1}^{*}$ and $\sum_{1}^{* *}$ denote the sum over all pairs of $m$-tuples $\left(i_{1}, \ldots, i_{m}\right)$, $\left(j_{1}, \ldots, j_{m}\right) \in C_{n, m}$ having all indices different and at least one ordered index equal, respectively, and $h_{1}$ is defined in (2.14). We further write

$$
Q_{n}=\left(\begin{array}{c}
n \\
2 m
\end{array}\right)^{-1} \sum_{C_{n, 2 m}} h_{1 n}\left(X_{k_{1}}, \ldots, X_{k_{2 m}}\right),
$$

where

$$
h_{1 n}\left(X_{k_{1}}, \ldots, X_{k_{2 m}}\right)=\left(\begin{array}{c}
n \\
2 m
\end{array}\right)\left(\begin{array}{c}
n \\
m
\end{array}\right)^{-2} \sum^{+} h_{1}\left(X_{i_{1}}, \ldots, X_{i_{m}}, X_{j_{1}} \ldots, X_{j_{m}}\right)
$$

and $\sum^{+}$denotes the sum over all $\left(\begin{array}{c}2 m \\ m\end{array}\right)$ summands $h_{1}\left(X_{i_{1}}, \ldots, X_{i_{m}}, X_{j_{1}} \ldots, X_{j_{m}}\right)$ with $\left\{i_{1}, \ldots, i_{m}, j_{1}, \ldots, j_{m}\right\}=\left\{k_{1}, \ldots, k_{2 m}\right\}$. Observe that

$$
\begin{aligned}
E & {\left[E\left(h_{1 n}\left(X_{1}, \ldots, X_{2 m}\right) \mid X_{1}, \ldots, X_{k}\right)\right]^{2} } \\
& =E\left(\left[E\left(\left(\begin{array}{c}
n \\
2 m
\end{array}\right)\left(\begin{array}{c}
n \\
m
\end{array}\right) \sum^{-2} h_{1}\left(X_{i_{1}}, \ldots, X_{i_{m}}, X_{j_{1}} \ldots, X_{j_{m}}\right) \mid X_{k}\right)\right]^{2}\right) \\
& =\left(\begin{array}{c}
n \\
2 m
\end{array}\right)^{2}\left(\begin{array}{c}
n \\
m
\end{array}\right)^{-4}\left(\begin{array}{c}
2 m \\
m
\end{array}\right)^{2} E\left[E\left(h_{1}\left(X_{1}, \ldots, X_{2 m}\right) \mid X_{1}, \ldots, X_{k}\right)\right]^{2} \\
& \leq E\left[E\left(h_{1}\left(X_{1}, \ldots, X_{2 m}\right) \mid X_{1}, \ldots, X_{k}\right)\right]^{2} .
\end{aligned}
$$

By Eq. (10.80) in [3], we have for $2 \leq k \leq 2 m$,

$$
E\left[E\left(h_{1}\left(X_{1}, \ldots, X_{2 m}\right) \mid X_{1}, \ldots, X_{k}\right)\right]^{2} \leq \frac{k}{2 m} \sigma_{3}^{2}
$$

and by some simple calculation, we have

$$
E\left[E\left(h_{1}\left(X_{1}, \ldots, X_{2 m}\right) \mid X_{1}\right)\right]=0,
$$

hence 


$$
\begin{aligned}
E Q_{n}^{2} & =\left(\begin{array}{c}
n \\
2 m
\end{array}\right)^{-1} \sum_{k=1}^{2 m}\left(\begin{array}{c}
2 m \\
k
\end{array}\right)\left(\begin{array}{c}
n-2 m \\
2 m-k
\end{array}\right) E\left[E\left(h_{1 n}\left(X_{1}, \ldots, X_{2 m}\right) \mid X_{1}, \ldots, X_{k}\right)\right]^{2} \\
& \leq\left(\begin{array}{c}
n \\
2 m
\end{array}\right) \sum_{k=2}^{-1}\left(\begin{array}{c}
2 m \\
k
\end{array}\right)\left(\begin{array}{c}
n-2 m \\
2 m-k
\end{array}\right) \frac{k}{2 m} \sigma_{3}^{2} \\
& \leq \frac{2 m(2 m-1)^{2}}{n(n-2 m+1)} \sigma_{3}^{2} \\
& \leq \frac{8 m^{3} \sigma_{0}^{2}}{n^{2}} .
\end{aligned}
$$

Next we give an upper bound of $E R_{n}^{2}$. Notice that the number of terms in $\sum_{1}^{* *}$ equals

$$
\begin{aligned}
\operatorname{Card}\left(\sum_{1}^{* *}\right) & =\left(\begin{array}{l}
n \\
m
\end{array}\right)^{2}-\left(\begin{array}{c}
n \\
m
\end{array}\right)\left(\begin{array}{c}
n-m \\
m
\end{array}\right) \\
& =\left(\begin{array}{c}
n \\
m
\end{array}\right)^{2}\left(1-\left(1-\frac{m}{n}\right)\left(1-\frac{m}{n-1}\right) \cdots\left(1-\frac{m}{n-m+1}\right)\right) \\
& \leq\left(\begin{array}{c}
n \\
m
\end{array}\right)^{2} m\left(\frac{1}{n}+\cdots+\frac{1}{n-m+1}\right) \\
& \leq\left(\begin{array}{c}
n \\
m
\end{array}\right)^{2} \frac{m^{2}}{(n-m+1)} \leq \frac{2 m^{2}}{n}\left(\begin{array}{c}
n \\
m
\end{array}\right)^{2} .
\end{aligned}
$$

Hence, by (2.16) and the Minkowski inequality

$$
E R_{n}^{2} \leq \frac{16 m^{4} \sigma_{0}^{2}}{n^{2}}
$$

Thus, by (2.22) and (2.23)

$$
\begin{aligned}
E B^{2} & \leq \frac{0.5 n c_{0}^{2}}{m^{2} \sigma_{2}^{2}} E\left(Q_{n}^{2}+R_{n}^{2}\right) \\
& \leq \frac{0.5 n c_{0}^{2}}{m^{2} \sigma_{2}^{2}}\left(\frac{8 m^{3} \sigma_{0}^{2}}{n^{2}}+\frac{16 m^{4} \sigma_{0}^{2}}{n^{2}}\right) \\
& \leq \frac{12 m^{2} c_{0}^{2} \sigma_{0}^{2}}{n \sigma_{2}^{2}}
\end{aligned}
$$

This proves (2.19).

\subsubsection{Proof of Lemma 2.4}

Let $\Delta_{l}=C+D$, where

$$
\begin{aligned}
& C=-\left(\begin{array}{c}
n \\
m
\end{array}\right)^{-1} \frac{\sqrt{n}}{m \sigma_{2}} \sum_{\substack{1 \leq i_{1}<\cdots<i_{m} \leq n, i_{j} \neq l, j \in\{1, \ldots, m\}}} \bar{A}_{m}\left(x_{i_{1}}, \ldots, x_{i_{m}}\right), \\
& D=-\frac{\sqrt{n}}{m \sigma_{2}} \int_{-\infty}^{+\infty}\left[\varphi\left(H_{n, l}(y)\right)-\varphi(H(y))-\left(H_{n, l}(y)-H(y)\right) J(H(y))\right] d y .
\end{aligned}
$$


Clearly, we have

$$
E\left(\Delta-\Delta_{l}\right)^{2} \leq 2 E(A-C)^{2}+2 E(B-D)^{2} .
$$

Note that

$$
\begin{aligned}
E(A-C)^{2}= & \left(-\left(\begin{array}{c}
n \\
m
\end{array}\right)^{-1} \frac{\sqrt{n}}{m \sigma_{2}}\right)^{2} E\left(\left(\sum_{1 \leq i_{1}<\cdots<i_{m} \leq n}-\sum_{\substack{1 \leq i_{1}<\cdots<i_{m} \leq n, i_{j} \neq l, j \in\{1, \ldots, m\}}}\right)\right. \\
& \left.\times \bar{A}_{m}\left(x_{i_{1}}, \ldots, x_{i_{m}}\right)\right)^{2} .
\end{aligned}
$$

Following the proof in [3, p. 287] and similarly to (2.18) we have

$$
\begin{aligned}
E(A-C)^{2} & \leq \frac{2(m-1)^{2} \sigma^{2}}{n m(n-m+1) \sigma_{2}^{2}} \\
& \leq \frac{2(m-1)^{2}\left(|J(0)|+c_{0}\right)^{2} \sigma_{0}^{2}}{n m(n-m+1) \sigma_{2}^{2}} \\
& \leq \frac{4 m\left(|J(0)|+c_{0}\right)^{2} \sigma_{0}^{2}}{n^{2} \sigma_{2}^{2}} .
\end{aligned}
$$

To complete the proof of (2.11), it suffices to show that

$$
E(B-D)^{2} \leq \frac{30 m^{2} c_{0}^{2} \sigma_{0}^{2}}{n^{2} \sigma_{2}^{2}} .
$$

Let

$$
\begin{aligned}
L & =\int_{-\infty}^{+\infty}\left[\varphi\left(H_{n}(y)\right)-\varphi(H(y))-\left(H_{n}(y)-H(y)\right) J(H(y))\right] d y, \\
L_{l} & =\int_{-\infty}^{+\infty}\left[\varphi\left(H_{n, l}(y)\right)-\varphi(H(y))-\left(H_{n, l}(y)-H(y)\right) J(H(y))\right] d y .
\end{aligned}
$$

Similarly to (2.21), we have

$$
\begin{aligned}
& \frac{m^{2} \sigma_{2}^{2}}{n} E(B-D)^{2} \\
& =E\left(\int_{-\infty}^{+\infty}\left[\varphi\left(H_{n}(y)\right)-\varphi\left(H_{n, l}(y)\right)-\left(H_{n}(y)-H_{n, l}(y)\right) J\left(H_{n, l}(y)\right)\right] d y\right. \\
& \left.\quad+\int_{-\infty}^{+\infty}\left(H_{n}(y)-H_{n, l}(y)\right)\left[J\left(H_{n, l}(y)\right)-J(H(y))\right] d y\right)^{2} \\
& \leq c_{0}^{2} E\left(0.5 \int_{-\infty}^{+\infty}\left(H_{n}(y)-H_{n, l}(y)\right)^{2} d y\right. \\
& \left.\quad+\int_{-\infty}^{+\infty}\left|H_{n}(y)-H_{n, l}(y)\right|\left|H_{n, l}(y)-H(y)\right| d y\right)^{2}
\end{aligned}
$$




$$
\begin{aligned}
\leq & 0.5 c_{0}^{2} E\left(\int_{-\infty}^{+\infty}\left(H_{n}(y)-H_{n, l}(y)\right)^{2} d y\right)^{2} \\
& +2 c_{0}^{2} E\left(\int_{-\infty}^{+\infty}\left|H_{n}(y)-H_{n, l}(y)\right|\left|H_{n, l}(y)-H(y)\right| d y\right)^{2} \\
= & 0.5 c_{0}^{2} L+2 c_{0}^{2} L_{l},
\end{aligned}
$$

where

$$
\begin{aligned}
L & =E\left(\int_{-\infty}^{+\infty}\left(H_{n}(y)-H_{n, l}(y)\right)^{2} d y\right)^{2}, \\
L_{l} & =E\left(\int_{-\infty}^{+\infty}\left|H_{n}(y)-H_{n, l}(y)\right|\left|H_{n, l}(y)-H(y)\right| d y\right)^{2} .
\end{aligned}
$$

We first consider $L$. By (1.10) and (2.9),

$$
\begin{aligned}
& \int_{-\infty}^{+\infty}\left(H_{n}(y)-H_{n, l}(y)\right)^{2} d y \\
& =\left(\begin{array}{l}
n \\
m
\end{array}\right)^{-2} \int_{-\infty}^{+\infty}\left(\sum_{1 \leq i_{1}<\cdots<i_{m-1} \leq n-1}\left(I\left[h\left(X_{i_{1}}, \ldots, X_{i_{m-1}}, X_{n}\right) \leq y\right]-H(y)\right)\right)^{2} d y \\
& \quad=\left(\begin{array}{c}
n \\
m
\end{array}\right)^{-2} \sum_{2} h_{2}\left(X_{i_{1}}, \ldots, X_{i_{m-1}}, X_{j_{1}}, \ldots, X_{j_{m-1}}, X_{n}\right),
\end{aligned}
$$

where $\sum_{2}$ denotes the sum over all pairs of $m-1$-tuples $\left(i_{1}, \ldots, i_{m-1}\right),\left(j_{1}, \ldots\right.$, $\left.j_{m-1}\right) \in C_{n-1, m-1}$ and

$$
\begin{aligned}
& h_{2}\left(x_{1}, \ldots, x_{m-1}, x_{m}, \ldots, x_{2 m-2}, x_{n}\right) \\
& =\int_{-\infty}^{+\infty}\left(I\left[h\left(x_{1}, \ldots, x_{m-1}, x_{n}\right) \leq y\right]-H(y)\right) \\
& \quad \times\left(I\left[h\left(x_{m}, \ldots, x_{2 m-2}, x_{n}\right) \leq y\right]-H(y)\right) d y .
\end{aligned}
$$

A direct calculation gives $\operatorname{Card}\left(\sum_{2}\right)=\left(\begin{array}{c}n-1 \\ m-1\end{array}\right)^{2}$. So, by (2.16) and the Minkowski inequality, we obtain

$$
L \leq \frac{4 m^{4} \sigma_{0}^{2}}{n^{4}}
$$

As to $L_{l}$, note that

$$
\begin{aligned}
& \int_{-\infty}^{+\infty}\left|H_{n}(y)-H_{n, l}(y)\right|\left|H_{n, l}(y)-H(y)\right| d y \\
& =\left(\begin{array}{c}
n \\
m
\end{array}\right)^{-2} \int_{-\infty}^{+\infty}\left|\sum_{1 \leq i_{1}<\cdots<i_{m-1} \leq n-1}\left(I\left[h\left(X_{i_{1}}, \ldots, X_{i_{m-1}}, X_{n}\right) \leq y\right]-H(y)\right)\right| \\
& \quad \times\left|\sum_{1 \leq j_{1}<\cdots<j_{m} \leq n-1}\left(I\left[h\left(X_{j_{1}}, \ldots, X_{j_{m}}\right) \leq y\right]-H(y)\right)\right| d y
\end{aligned}
$$




$$
\begin{aligned}
& \leq\left(\begin{array}{c}
n \\
m
\end{array}\right)^{-2} \sum_{1 \leq i_{1}<\cdots<i_{m-1} \leq n-1} \int_{-\infty}^{+\infty}\left|I\left[h\left(X_{i_{1}}, \ldots, X_{i_{m-1}}, X_{n}\right) \leq y\right]-H(y)\right| \\
& \quad \times\left|\sum_{1 \leq j_{1}<\cdots<j_{m} \leq n-1}\left(I\left[h\left(X_{j_{1}}, \ldots, X_{j_{m}}\right) \leq y\right]-H(y)\right)\right| d y .
\end{aligned}
$$

Thus, by the Minkowski inequality again

$$
\begin{aligned}
L_{l} \leq & \left(\begin{array}{c}
n \\
m
\end{array}\right)^{-4}\left(\begin{array}{c}
n-1 \\
m-1
\end{array}\right)^{2} E\left(\int_{-\infty}^{\infty}\left|I\left[h\left(X_{1}, \ldots, X_{m-1}, X_{n}\right) \leq y\right]-H(y)\right|\right. \\
& \left.\times\left|\sum_{1 \leq j_{1}<\cdots<j_{m} \leq n-1}\left(I\left[h\left(X_{j_{1}}, \ldots, X_{j_{m}}\right) \leq y\right]-H(y)\right)\right| d y\right)^{2} \\
\leq & L_{l, 1}+L_{l, 2},
\end{aligned}
$$

where

$$
\begin{aligned}
L_{l, 1}= & \frac{2 m^{2}}{n^{2}}\left(\begin{array}{c}
n \\
m
\end{array}\right)^{-2} E\left(\int_{-\infty}^{\infty}\left|I\left[h\left(X_{1}, \ldots, X_{m-1}, X_{n}\right) \leq y\right]-H(y)\right|\right. \\
& \left.\times\left|\sum_{3}\left(I\left[h\left(X_{j_{1}}, \ldots, X_{j_{m}}\right) \leq y\right]-H(y)\right)\right| d y\right)^{2}, \\
L_{l, 2}= & \frac{2 m^{2}}{n^{2}}\left(\begin{array}{l}
n \\
m
\end{array}\right)^{-2} E\left(\int_{-\infty}^{\infty}\left|I\left[h\left(X_{1}, \ldots, X_{m-1}, X_{n}\right) \leq y\right]-H(y)\right|\right. \\
& \left.\times\left|\sum_{4}\left(I\left[h\left(X_{j_{1}}, \ldots, X_{j_{m}}\right) \leq y\right]-H(y)\right)\right| d y\right)^{2},
\end{aligned}
$$

$\sum_{3}$ denotes the sum over $\left(j_{1}, \ldots, j_{m}\right) \in C_{n-1, m}-\{1, \ldots, m-1\}$ and $\sum_{4}$ denotes the sum over all pairs $\left(i_{1}, \ldots, i_{m-1}, n\right) \in C_{n-1, m-1}$ and $\left\{j_{1}, \ldots, j_{m-1}\right\} \cap\{1, \ldots$, $m-1\} \neq \emptyset$. Observing that

$$
\operatorname{Card}\left(\sum_{4}\right)=\left(\begin{array}{c}
n-1 \\
m
\end{array}\right)-\left(\begin{array}{c}
n-m \\
m
\end{array}\right) \leq \frac{2 m}{n}\left(\begin{array}{c}
n-1 \\
m
\end{array}\right)
$$

we have

$$
\begin{aligned}
L_{l, 2} \leq & \frac{4 m^{3}}{n^{3}}\left(\begin{array}{c}
n \\
m
\end{array}\right)^{-2}\left(\begin{array}{c}
n-1 \\
m
\end{array}\right) \sum_{4} E\left(\int_{-\infty}^{\infty}\left|I\left[h\left(X_{1}, \ldots, X_{m-1}, X_{n}\right) \leq y\right]-H(y)\right|\right. \\
& \left.\times\left|I\left[h\left(X_{j_{1}}, \ldots, X_{j_{m}}\right) \leq y\right]-H(y)\right| d y\right)^{2} \\
\leq & \frac{32 m^{4}}{n^{4}}\left(\begin{array}{c}
n \\
m
\end{array}\right)^{-2}\left(\begin{array}{c}
n-1 \\
m
\end{array}\right){ }^{2} \sigma_{0}^{2} \\
\leq & \frac{32 m^{4}}{n^{4}} \sigma_{0}^{2} \leq \frac{8 m^{4} \sigma_{0}^{2}}{n^{3}} .
\end{aligned}
$$

In the second inequality above, we used (2.16).

To estimate $L_{l, 1}$, note that 


$$
\begin{aligned}
& E\left(\int_{-\infty}^{\infty}\left|I\left[h\left(X_{1}, \ldots, X_{m-1}, X_{n}\right) \leq y\right]-H(y)\right|\right. \\
& \left.\times\left|\sum_{3}\left(I\left[h\left(X_{j_{1}}, \ldots, X_{j_{m}}\right) \leq y\right]-H(y)\right)\right| d y\right)^{2} \\
& =E\left(\int_{-\infty}^{\infty} \int_{-\infty}^{\infty}\left|I\left[h\left(X_{1}, \ldots, X_{m-1}, X_{n}\right) \leq x\right]-H(x)\right|\right. \\
& \times\left|I\left[h\left(X_{1}, \ldots, X_{m-1}, X_{n}\right) \leq y\right]-H(y)\right| \\
& \times\left|\sum_{3}\left(I\left[h\left(X_{j_{1}}, \ldots, X_{j_{m}}\right) \leq x\right]-H(x)\right)\right| \\
& \left.\times\left|\sum_{3}\left(I\left[h\left(X_{j_{1}}, \ldots, X_{j_{m}}\right) \leq y\right]-H(y)\right)\right|\right) d x d y \\
& =\int_{-\infty}^{\infty} \int_{-\infty}^{\infty} E\left(\left|I\left[h\left(X_{1}, \ldots, X_{m-1}, X_{n}\right) \leq x\right]-H(x)\right|\right. \\
& \left.\times\left|I\left[h\left(X_{1}, \ldots, X_{m-1}, X_{n}\right) \leq y\right]-H(y)\right|\right) \\
& \times E\left(\left|\sum_{3}\left(I\left[h\left(X_{j_{1}}, \ldots, X_{j_{m}}\right) \leq x\right]-H(x)\right)\right|\right. \\
& \left.\times\left|\sum_{3}\left(I\left[h\left(X_{j_{1}}, \ldots, X_{j_{m}}\right) \leq y\right]-H(y)\right)\right|\right) d x d y \\
& \leq \int_{-\infty}^{\infty} \int_{-\infty}^{\infty}\left\|I\left[h\left(X_{1}, \ldots, X_{m-1}, X_{n}\right) \leq x\right]-H(x)\right\|_{2} \\
& \times\left\|I\left[h\left(X_{1}, \ldots, X_{m-1}, X_{n}\right) \leq y\right]-H(y)\right\|_{2} \\
& \times\left\|\sum_{3}\left(I\left[h\left(X_{j_{1}}, \ldots, X_{j_{m}}\right) \leq x\right]-H(x)\right)\right\|_{2} \\
& \times\left\|\sum_{3}\left(I\left[h\left(X_{j_{1}}, \ldots, X_{j_{m}}\right) \leq y\right]-H(y)\right)\right\|_{2} d x d y \\
& \leq \int_{-\infty}^{\infty} \int_{-\infty}^{\infty}(H(x)(1-H(x)))^{1 / 2}(H(y)(1-H(y)))^{1 / 2} \\
& \times \frac{3 m^{2}}{n}\left(\begin{array}{c}
n-m \\
m
\end{array}\right)^{2}(H(x)(1-H(x)))^{1 / 2}(H(y)(1-H(y)))^{1 / 2} d x d y \\
& \leq \frac{3 m^{2}}{n}\left(\begin{array}{c}
n-m \\
m
\end{array}\right)^{2} \sigma_{0}^{2} \text {. }
\end{aligned}
$$

Here we used the following estimate:

$$
\left\|\sum_{3}\left(I\left[h\left(X_{j_{1}}, \ldots, X_{j_{m}}\right) \leq x\right]-H(x)\right)\right\|_{2}^{2} \leq \frac{3 m^{2}}{n}\left(\begin{array}{c}
n-m \\
m
\end{array}\right)^{2} H(x)(1-H(x)) .
$$


Thus, we obtain

$$
L_{l, 1} \leq \frac{6 m^{4} \sigma_{0}^{2}}{n^{3}}
$$

and hence

$$
L_{l} \leq \frac{14 m^{4} \sigma_{0}^{2}}{n^{3}}
$$

by (2.27) and (2.28).

This proves (2.24) by (2.25), (2.26), and (2.30), as desired. This completes the proof of Lemma 2.4 .

Acknowledgements The research of Q.-M. Shao is partly supported by Hong Kong RGC GRF 603710, 2130344.

\section{References}

1. Borovshikh, Y.V., Weber, N.C.: Asymptotic distributions for a class of generalized $L$-statistics. Bernoulli 16, 1177-1190 (2010)

2. Cai, Z.W.: Moderate deviations and large deviations of generalized L-statistics. Chin. Ann. Math., Ser. A 13, 364-372 (1992)

3. Chen, L.H.Y., Goldstein, L., Shao, Q.M.: Normal Approximation by Stein's Method. Springer, Heidelberg (2011)

4. Chen, L.H.Y., Shao, Q.M.: Normal approximation for nonlinear statistics using a concentration inequality approach. Bernoulli 13, 581-599 (2007)

5. Helmers, R., Janssen, P., Serfling, R.J.: Berry-Esseen bound and bootstrap results for generalized L-statistics. Scand. J. Stat. 17, 65-77 (1990)

6. Helmers, R., Ruymgaart, F.H.: Asymptotic normality of generalized L-statistics with unbounded scores. J. Stat. Plan. Inference 19, 43-53 (1988)

7. Major, P.: Asymptotic distributions for weighted U-statistics. Ann. Probab. 22, 1514-1535 (1994)

8. O’Neil, K.A., Redner, R.A.: Asymptotic distributions of weighted U-statistics of degree two. Ann. Probab. 21, 1159-1169 (1993)

9. Rifi, M., Utzet, F.: On the asymptotic behavior of weighted U-statistics. J. Theor. Probab. 13, 141-167 (2000)

10. Shapiro, C.P., Hubert, L.: Asymptotic normality of permutation statistics derived from weighted sums of bivariate functions. Ann. Stat. 7, 788-794 (1979)

11. Serfling, R.: Approximation Theorems of Mathematical Statistics. Wiley, New York (1980)

12. Serfling, R.: Generalized $L-, M$ - and $R$-statistics. Ann. Stat. 12, 76-86 (1984)

13. Serfling, R.: Robust estimation via generalized L-statistics: theory, applications, and perspectives. In: Advances on Methodological and Applied Aspects of Probability and Statistics pp. 197-217. Taylor \& Francis, London (1998) 